\title{
An Improvement for Fuzzy Stochastic Goal Programming Problems
}

\author{
Shu-Cheng Lin, ${ }^{1}$ Han-Wen Tuan, ${ }^{2}$ and Peterson Julian ${ }^{3}$ \\ ${ }^{1}$ Department of Hotel Management, Lee-Ming Institute of Technology, New Taipei, Taiwan \\ ${ }^{2}$ Department of Computer Science and Information Management, Hungkuang University, Taichung, Taiwan \\ ${ }^{3}$ Department of Traffic Science, Central Police University, Taoyuan, Taiwan
}

Correspondence should be addressed to Peterson Julian; petersonjulian44328@gmail.com

Received 2 January 2017; Revised 5 June 2017; Accepted 11 June 2017; Published 25 July 2017

Academic Editor: Leonid Shaikhet

Copyright (C) 2017 Shu-Cheng Lin et al. This is an open access article distributed under the Creative Commons Attribution License, which permits unrestricted use, distribution, and reproduction in any medium, provided the original work is properly cited.

\begin{abstract}
We examined the solution process for linear programming problems under a fuzzy and random environment to transform fuzzy stochastic goal programming problems into standard linear programming problems. A previous paper that revised the solution process with the lower-side attainment index motivated our work. In this paper, we worked on a revision for both-side attainment index to amend its definition and theorems. Two previous examples were used to examine and demonstrate our improvement over previous results. Our findings not only improve the previous paper with both-side attainment index, but also provide a theoretical extension from lower-side attainment index to the both-side attainment index.
\end{abstract}

\section{Introduction}

Except for a very small portion of events that are predictable and controllable, most of market-, demand-, and financerelated and other activities possess the features of fuzziness and randomness [1]. In order to construct a more realistic inventory model to deal with fuzziness and randomness in the business environment, scores of academicians and practitioners have been placing emphasis on the associated issues. Research engaging in various fuzzy and random mathematical programming techniques includes Mahalingam et al. [2]. One of the major approaches is to construct the mathematical models where the defuzzifying and derandomizing processes are fulfilled. A general deterministic linear programming (LP) model can then be formulated and the uncertainties can be eliminated [3]. By way of development, the so-called ordering measures of fuzzy numbers have also attracted attention to some extent by researchers in such areas as the compensation method [4], the signed distance method [5], the expected mid-point approach [6], and so on. Zimmermann [7] solved the LP problems with fuzzy environment. He employed the max-min tolerance method, with the criterion of the highest membership degree, to convert the fuzzy LP into crisp LP. The crisp LP can then be solved through traditional solution methods. Afterwards, scores of relevant studies have made efforts and have contributed solutions with varying degrees of success. As far as the progress of this area is concerned, two methods are well known as the primary ways of tackling the problems with a fuzzy and random environment. The sequential approach is used to carry out the defuzzifying and derandomizing processes, one at a time [8]. A simultaneous approach is used to fulfill the relaxation of fuzzy and random constraints [7]. However, the current use of both approaches has produced bottlenecks in either complexity or workload. Should the sequential manner be adopted, a great deal of additional variables and constraints would be generated while carrying out the defuzzifying and derandomizing processes. On the other hand, should the simultaneous manner be employed, the calculation of the expected value is quite cumbersome while trying to accomplish the task of removing the fuzzy and stochastic factors.

Besides the sequential and simultaneous approaches, other trials have also been conducted and confirmed. For instance, instead of using the defuzzifying and derandomizing processes, Liu [8] offered a theoretical framework with a hybrid intelligent algorithm to directly tackle the fuzzy random dependent-chance programming models. Through 
a semi-infinite optimization, Luhandjula [9] tried to convert the original LP in the presence of fuzzy random variable coefficients into a stochastic program so that the techniques of stochastic optimization can be applied.

Even though there have been at least two papers, Fei [10] and $\mathrm{Xu}$ and Liu [11], that have referred to Hop [12], the theoretical problem of Hop [12] and the questionable result in the numerical example of Hop [12] were not discovered nor addressed.

Moreover, our paper not only revises the questionable results in Hop [13], but also extends Hop [13] and Chou et al. [14]. For two fuzzy numbers $\widetilde{P}$ and $\widetilde{Q}$, in Hop [13] and Chou et al. [14], they only considered, $D(\widetilde{P}, \widetilde{Q})$, the lowerside attainment index of $\widetilde{P}$ to $\widetilde{Q}$ such that, in their definition, they assumed that $\widetilde{P} \leq \widetilde{Q}$. It means that if $\widetilde{P}$ and $\widetilde{Q}$ are two triangular fuzzy numbers, with $\widetilde{P}=(u, a, b)$ and $\widetilde{Q}=(v, c, d)$, then they assumed that $u \leq v$.

However, in Hop [12], he considered, $V(\widetilde{P}, \widetilde{Q})$, the bothside attainment index of two fuzzy numbers $\widetilde{P}$ and $\widetilde{Q}$, where $V(\widetilde{P}, \widetilde{Q})=\min \{D(\widetilde{P}, \widetilde{Q}), D(\widetilde{Q}, \widetilde{P})\}$. For the well defined $V(\widetilde{P}, \widetilde{Q})$, we must remove the condition of $u \leq v$ where $\widetilde{P}=(u, a, b)$ and $\widetilde{Q}=(v, c, d)$, when we define $D(\widetilde{P}, \widetilde{Q})$.

The rest of this paper is organized as follows. Section 2 is the brief review of the main definitions, theorems, and propositions of Hop [12]. Section 3 provides our findings, revision, and improvement in response to the questionable theoretical derivation and analytical arguments Hop had presented. Section 4 provides a numerical example to show the practical solution-finding algorithm of our approach and a comparison between Hop's results and ours.

\section{Review of Previous Results}

In the following, a brief review of Hop's approach [12] is provided as the basis and motivation for our revision. The environment in which the problem and the approach reside is first defined as follows. After the fuzzy variable is defined, a measure for a pair of such variables is defined next in order to indicate the degree of conformance to the inequality relationship between them.

Definition 1 of Hop [12]. The lower-side attainment index of fuzzy random variable $\widetilde{\bar{P}}$ to fuzzy random variable $\widetilde{\bar{Q}}$, under the condition $\widetilde{\bar{P}} \leq \widetilde{\bar{Q}}$ (which will be removed after our improvement), is defined as

$$
\begin{aligned}
& D(\widetilde{\bar{P}}, \widetilde{\bar{Q}})=\int_{0}^{1} \max \left\{0, \sup \left\{s \in R: \widetilde{P}_{w}(s) \geq \alpha\right\}\right. \\
& \left.\quad-\inf \left\{r \in R: \widetilde{Q}_{w}(r) \geq \alpha\right\}\right\} \mathrm{d} \alpha .
\end{aligned}
$$

An extension of $D(\widetilde{\bar{P}}, \widetilde{\bar{Q}})$ measure is the new concept of the both-side attainment index introduced by Hop [12].
Definition 2 of Hop [12]. The both-side attainment index of fuzzy random variable $\widetilde{\bar{P}}$ to fuzzy random variable $\widetilde{\bar{Q}}$ is defined as

$$
V(\widetilde{\widetilde{P}}, \widetilde{\bar{Q}})=\max \{0, \min (D(\widetilde{\bar{P}}, \widetilde{\bar{Q}}), D(\widetilde{\bar{Q}}, \widetilde{\bar{P}}))\} .
$$

Hop [12] assumed that $\{\tilde{t}: \tilde{t}=(t, a, b), a, b \geq 0\}$ is the collection of triangular fuzzy numbers with the following membership function:

$$
\mu_{\tilde{t}}(x)= \begin{cases}\max \left\{0,1-\frac{t-x}{a}\right\}, & \text { if } x \leq t, \\ \max \left\{0,1-\frac{x-t}{b}\right\}, & \text { if } x \geq t,\end{cases}
$$

where the scalars $a, b \geq 0$ are called the left and right spreads, respectively. For $0 \leq \alpha \leq 1$, Hop [12] let

$$
\begin{aligned}
& P_{\alpha}^{l}(w)=\inf \left\{x \in R: \widetilde{P}_{w}(x) \geq \alpha\right\}, \\
& P_{\alpha}^{u}(w)=\sup \left\{x \in R: \widetilde{P}_{w}(x) \geq \alpha\right\} .
\end{aligned}
$$

The $\alpha$-cut of the fuzzy set $\widetilde{P}_{w}$ is defined as

$$
\widetilde{P}_{w}^{\alpha}=\left[P_{\alpha}^{l}(w), P_{\alpha}^{u}(w)\right] .
$$

According to Theorem 1 of Luhandjula [9], if $\widetilde{P}_{w}, \widetilde{Q}_{w} \in T$, then Hop [12] assumed that

$$
\begin{aligned}
\widetilde{P}_{w} & =(u(w), a(w), b(w)), \\
\widetilde{Q}_{w} & =(v(w), c(w), d(w))
\end{aligned}
$$

and the $\alpha$-cut of $\widetilde{P}_{w}$ is

$$
\widetilde{P}_{w}^{\alpha}=[u(w)-(1-\alpha) a(w), u(w)+(1-\alpha) b(w)] .
$$

The lower-side attainment index of $\widetilde{\bar{P}}$ to $\widetilde{\bar{Q}}$ at $\alpha$-level is

$$
D(\widetilde{\bar{P}}, \widetilde{\bar{Q}})_{\alpha}=\max \left\{0, P_{\alpha}^{u}(w)-Q_{\alpha}^{l}(w)\right\} .
$$

Hop [12] developed the main result in the following proposition.

Proposition 1 of Hop [12]. Consider two triangular fuzzy random numbers $\widetilde{\bar{P}}$ and $\widetilde{\bar{Q}}, \widetilde{\bar{P}} \leq \widetilde{\bar{Q}}$; the average lower-side attainment of $\stackrel{\widetilde{P}}{\text { to }} \widetilde{\bar{Q}}$ is

$$
\bar{D}(\widetilde{\bar{P}}, \widetilde{\bar{Q}})=\frac{u(w)-v(w)+b(w)+c(w)}{2} .
$$

Proposition 1 is proved as follows. Hop [12] first computed the integration of the lower-side attainment index of $\widetilde{\bar{P}}$ to $\widetilde{\bar{Q}}$ at $\alpha$-level for $0 \leq \alpha \leq 1$.

$$
\begin{aligned}
& \bar{D}(\widetilde{\bar{P}}, \widetilde{\bar{Q}})=\int_{0}^{1} D(\widetilde{\bar{P}}, \widetilde{\bar{Q}})_{\alpha} \mathrm{d} \alpha \\
& \quad=\int_{0}^{\lambda^{*}=1-(v(w)-u(w)) /(b(w)+c(w))}[u(w)-v(w) \\
& \quad+(1-\alpha)(b(w)+c(w))] \mathrm{d} \alpha .
\end{aligned}
$$


He then considered the average lower-side attainment of $\widetilde{\bar{P}}$ to $\widetilde{\bar{Q}}$ as

$$
\bar{D}(\widetilde{\bar{P}}, \widetilde{\bar{Q}})=\frac{1}{\lambda^{*}} \int_{0}^{1} D(\widetilde{\bar{P}}, \widetilde{\bar{Q}})_{\alpha} \mathrm{d} \alpha
$$

After lengthy computation, he derived his Proposition 1.

Moreover, from (2) and (9), Hop [12] assumed that the average both-side attainment index of $\widetilde{\bar{P}}$ to $\widetilde{\bar{Q}}$ is defined as

$$
\bar{V}(\widetilde{\bar{P}}, \widetilde{\bar{Q}})=\max \left\{0, \min \left\{\frac{u(w)-v(w)+b(w)+c(w)}{2}, \frac{v(w)-u(w)+a(w)+d(w)}{2}\right\}\right\} .
$$

From (12), he mentioned the following result.

Proposition 2 of Hop [12]. Let $\widetilde{\bar{P}}, \widetilde{\bar{Q}}, 0<\lambda(w), w \in \Omega$. Then $\bar{V}(\widetilde{\bar{P}}, \widetilde{\bar{Q}}) \geq \lambda(w)$ if and only if

$$
\begin{aligned}
& \bar{D}(\widetilde{\bar{P}}, \widetilde{\bar{Q}})=\frac{u(w)-v(w)+b(w)+c(w)}{2} \geq \lambda(w), \\
& \bar{D}(\widetilde{\bar{Q}}, \widetilde{\bar{P}})=\frac{v(w)-u(w)+a(w)+d(w)}{2} \geq \lambda(w) .
\end{aligned}
$$
(12).

Hop [12] claimed that the proof follows immediately from

\section{Our Improvement}

Recall the definition of the both-side attainment index in (2). If we followed the inequality condition of Hop [12] in Definition 1 , that is, $\widetilde{\bar{P}} \leq \widetilde{\bar{Q}}$, will be needed to validate the notion of $D(\widetilde{\bar{P}}, \widetilde{\bar{Q}})$. On the other hand, it would imply that $D(\widetilde{\bar{Q}}, \widetilde{\bar{P}})$ would only be valid under the condition of $\widetilde{\bar{Q}} \leq \widetilde{\bar{P}}$. In the definition where the both-side attainment index is valid, both $D(\widetilde{\bar{P}}, \widetilde{\bar{Q}})$ and $D(\widetilde{\bar{Q}}, \widetilde{\bar{P}})$ need to be computed, which, based on the definition in Hop [12], can only be realized if both $\widetilde{\bar{P}} \leq \widetilde{\bar{Q}}$ and $\widetilde{\bar{Q}} \leq \widetilde{\bar{P}}$ hold .

Here, we will provide an intuitive meaning for lowerside attainment index. $D(\widetilde{\bar{Q}}, \widetilde{\bar{P}})$ will compute the difference between the best case of $\widetilde{\bar{P}}$ and the worst case of $\widetilde{\bar{Q}}$ such that given a random variable $w$ and $\alpha$-cut, the integrand for the integration is $\max \left\{0,\left(\max \widetilde{P}_{w}^{\alpha}\right)-\left(\min \widetilde{Q}_{w}^{\alpha}\right)\right\}$. It is of no relevance to whether $\widetilde{\bar{P}} \leq \widetilde{\bar{Q}}$ or not. Hence, to make the lower-side attainment index correctly, the condition of $\widetilde{\bar{P}} \leq \widetilde{\bar{Q}}$ should be removed from Definition 1 . Moreover, because the lower-side attainment index as defined in (1) is related to the random variable $w$, therefore it should be related to $w$.

Since the lower-side attainment index of $\widetilde{\bar{P}}$ to $\widetilde{\bar{Q}}$ at $\alpha$-level is related to $w$ we may revise (8) as follows:

$$
D(\widetilde{\bar{P}}, \widetilde{\bar{Q}})_{\alpha}(w)=\max \left\{0, P_{\alpha}^{u}(w)-Q_{\alpha}^{l}(w)\right\}
$$

Therefore, the lower-side attainment index of fuzzy random variable $\widetilde{\bar{P}}$ to fuzzy random variable $\widetilde{\bar{Q}}$, without the unnecessary condition $\widetilde{\bar{P}} \leq \widetilde{\bar{Q}}$, is defined as

$$
D(\widetilde{\bar{P}}, \widetilde{\bar{Q}})(w)=\int_{0}^{1} D(\widetilde{\bar{P}}, \widetilde{\bar{Q}})_{\alpha}(w) \mathrm{d} \alpha
$$

Moreover, we reconsider the average lower-side attainment index of fuzzy random variable $\widetilde{\bar{P}}$ to $\widetilde{\bar{Q}}$, say $\bar{D}(\widetilde{\bar{P}}, \widetilde{\bar{Q}})$, without the unnecessary condition $\widetilde{\bar{P}} \leq \widetilde{\bar{Q}}$, which can be defined as follows:

$$
\begin{aligned}
& \text { If } D(\tilde{\bar{P}}, \widetilde{\bar{Q}})>0, \\
& \bar{D}(\tilde{\bar{P}}, \widetilde{\bar{Q}})=\frac{1}{\text { maximum height of intersected area }} D(\widetilde{\bar{P}}, \widetilde{\bar{Q}}), \\
& \text { if } D(\tilde{\bar{P}}, \widetilde{\bar{Q}})=0, \quad \bar{D}(\tilde{\bar{P}}, \widetilde{\bar{Q}})=0 .
\end{aligned}
$$

For a random variable $w$, from (6), $\widetilde{P}_{w}=(u(w), a(w), b(w))$ and $\widetilde{Q}_{w}=(v(w), c(w), d(w))$ are two fuzzy triangular numbers. If we try to consider the cases for the intersection between these two triangles, then we have to examine the relations among the following four points: $v(w)-c(w), v(w)+$ $d(w), u(w)-a(w)$, and $u(w)+b(w)$. From permutation, those are $4 !=24$ different results. However, owing to $v(w)-c(w)$ being the left end point of the fuzzy triangle and $v(w)+d(w)$ being the right end point, so $v(w)-c(w) \leq v(w)+d(w)$ must hold. Similarly, we also know that $u(w)-a(w) \leq u(w)+b(w)$, so there are $4 ! / 2 ! 2 !=6$ different results left. Moreover, we consider the relation of $u(w)>v(w)$ and $u(w) \leq v(w)$ to imply the following 10 cases as follows.

Case 1. $u(w)-a(w) \leq v(w)-c(w) \leq u(w)+b(w) \leq v(w)+$ $d(w) ; u(w) \leq v(w)$

Case 2. $u(w)-a(w) \leq v(w)-c(w) \leq u(w)+b(w) \leq v(w)+$ $d(w) ; u(w)>v(w)$.

Case 3. $u(w)-a(w) \leq v(w)-c(w) \leq v(w)+d(w) \leq u(w)+$ $b(w) ; u(w) \leq v(w)$.

Case 4. $u(w)-a(w) \leq v(w)-c(w) \leq v(w)+d(w) \leq u(w)+$ $b(w) ; u(w)>v(w)$.

Case 5. $u(w)-a(w) \leq u(w)+b(w)<v(w)-c(w) \leq v(w)+$ $d(w) ; u(w)<v(w)$. 
Case 6. $v(w)-c(w) \leq u(w)-a(w) \leq v(w)+d(w) \leq u(w)+$ $b(w) ; u(w)>v(w)$.

Case 7. $v(w)-c(w) \leq u(w)-a(w) \leq v(w)+d(w) \leq u(w)+$ $b(w) ; u(w) \leq v(w)$.

Case 8. $v(w)-c(w) \leq v(w)+d(w) \leq u(w)-a(w) \leq u(w)+$ $b(w) ; v(w) \leq u(w)$.

Case 9. $v(w)-c(w) \leq u(w)-a(w) \leq u(w)+b(w) \leq v(w)+$ $d(w) ; u(w)>v(w)$.

Case 10. $v(w)-c(w) \leq u(w)-a(w) \leq u(w)+b(w) \leq v(w)+$ $d(w) ; u(w) \leq v(w)$.

We first list them in Figures 1(a)-1(j), by two figures in one row, and then begin to consider the average lower-side attainment of $\widetilde{\bar{P}}$ to $\widetilde{\bar{Q}}$.

We divide the discussion into two parts:

For Case 5 with $u(w)+b(w)<v(w)-c(w)$ and for the rest of the Cases with $u(w)+b(w) \geq v(w)-c(w)$;

For Case 5, under the condition $u(w)+b(w)<v(w)-c(w)$, from

$$
\begin{aligned}
& P_{\alpha}^{u}(w)=u(w)+(1-\alpha) b(w) \leq u(w)+b(w), \\
& Q_{\alpha}^{l}(w)=v(w)-(1-\alpha) c(w) \geq v(w)-c(w),
\end{aligned}
$$

we can establish that $Q_{\alpha}^{l}(w)>P_{\alpha}^{u}(w)$. It follows that the lower-side attainment index at $\alpha$-level for this case is always zero, yielding $D(\widetilde{\bar{P}}, \widetilde{\bar{Q}})=0$ and then

$$
\bar{D}(\widetilde{\bar{P}}, \widetilde{\bar{Q}})=0
$$

This result is also self-evident by observing the graph in Figure 1(e).

For the rest of the nine cases, we consider that they are under the condition of $u(w)+b(w) \geq v(w)-c(w)$.

For Cases 1, 3, 7, and 10, the right wing of the membership function $\widetilde{P}_{w}$ with $y=1-(x(w)-u(w)) / b(w)$ for $u(w) \leq$ $x(w) \leq u(w)+b(w)$ and the left wing of the membership function $\widetilde{Q}_{w}$ with $y=1+(x(w)-v(w)) / c(w)$ for $v(w)-c(w) \leq$ $x(w) \leq v(w)$ have an intersection at

$$
\begin{aligned}
& \left(\frac{c(w) u(w)+b(w) v(w)}{b(w)+c(w)},\right. \\
& \left.\quad \frac{u(w)+b(w)-(v(w)-c(w))}{b(w)+c(w)}\right) .
\end{aligned}
$$

The $y$-coordinate of this intersection is denoted as $\lambda^{*}$ that is defined by Hop [12]. For Cases 1, 3, 7, and 10, we know that $u(w)+b(w) \geq v(w)-c(w)$ and $v(w) \geq u(w)$ are held such that

$$
0 \leq \lambda^{*}=\frac{u(w)+b(w)-(v(w)-c(w))}{b(w)+c(w)} \leq 1
$$

is satisfied. It yields that the shaded triangle in Figures 1(a), 1(c), 1(g), and 1(j), indicates the region that represents $P_{\alpha}^{u}(w) \geq Q_{\alpha}^{l}(w)$ so that, by (16), we know that

$$
\begin{aligned}
\bar{D}(\widetilde{\bar{P}}, \widetilde{\bar{Q}}) & =\frac{1}{2} \text { length of the width } \\
& =\frac{1}{2}[u(w)+b(w)-v(w)+c(w)] .
\end{aligned}
$$

That is, for Cases $1,3,7$, and $10, \bar{D}(\widetilde{\bar{P}}, \widetilde{\bar{Q}})$ can be obtained directly by taking a half of the distance between $(v(w)-$ $c(w), 0)$ and $(u(w)+b(w), 0)$, without referring to the value of $\lambda^{*}$. The purpose of discussing $\lambda^{*}$ is to explain that the overlapping region is a triangle.

For Cases 2, 4, 6, 8, and 9, owing to the condition $u(w)>$ $v(w)$, from

$$
\begin{aligned}
P_{\alpha}^{u}(w) & =u(w)+(1-\alpha) b(w) \geq u(w), \\
Q_{\alpha}^{l}(w) & =v(w)-(1-\alpha) c(w) \leq v(w),
\end{aligned}
$$

it yields that

$$
\begin{aligned}
P_{\alpha}^{u}(w) & =u(w)+(1-\alpha) b(w) \geq u(w)>v(w) \\
& \geq v(w)-(1-\alpha) c(w)=Q_{\alpha}^{l}(w)
\end{aligned}
$$

We know that

$$
\begin{aligned}
D(\widetilde{\bar{P}}, \widetilde{\bar{Q}})_{\alpha} & =\max \left\{0, P_{\alpha}^{u}(w)-Q_{\alpha}^{l}(w)\right\} \\
& =P_{\alpha}^{u}(w)-Q_{\alpha}^{l}(w) .
\end{aligned}
$$

We show that $Q_{\alpha}^{l}(w)<P_{\alpha}^{u}(w)$ for $0 \leq \alpha<1$ such that there is no intersection for the right wing of the membership function $\widetilde{P}_{w}$ and the left wing of the membership function $\widetilde{Q}_{w}$ so that the overlapping region becomes a trapezoid, with the maximum height being one. Using (16), it shows that

$$
\begin{aligned}
\bar{D}(\widetilde{\bar{P}}, \widetilde{\bar{Q}}) & =\text { area of the shaded trapezoid } \\
& =u(w)-v(w)+\frac{1}{2}[b(w)+c(w)] .
\end{aligned}
$$

Following our approach in (26), the complicated integration computation becomes unnecessary.

If we carefully examine the differences among the ten Cases we find that we may merge Cases 1, 3, 7, and 10 with the condition $u(w) \leq v(w)$. On the other hand, we may combine Cases 2, 4, 6, 8, and 9, together, with the condition $u(w)>$ $v(w)$. Moreover, for Case 5, from $u(w)+b(w)<v(w)-c(w)$, then $u(w) \leq v(w)$ holds. 


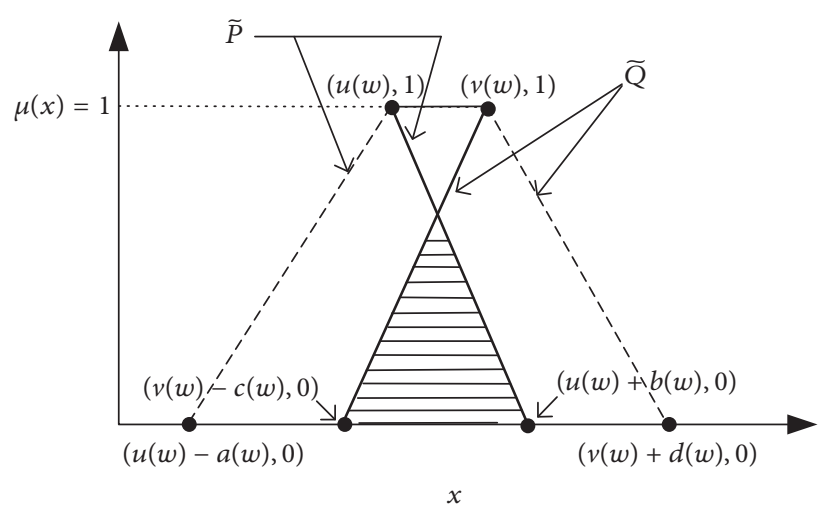

(a) Case 1

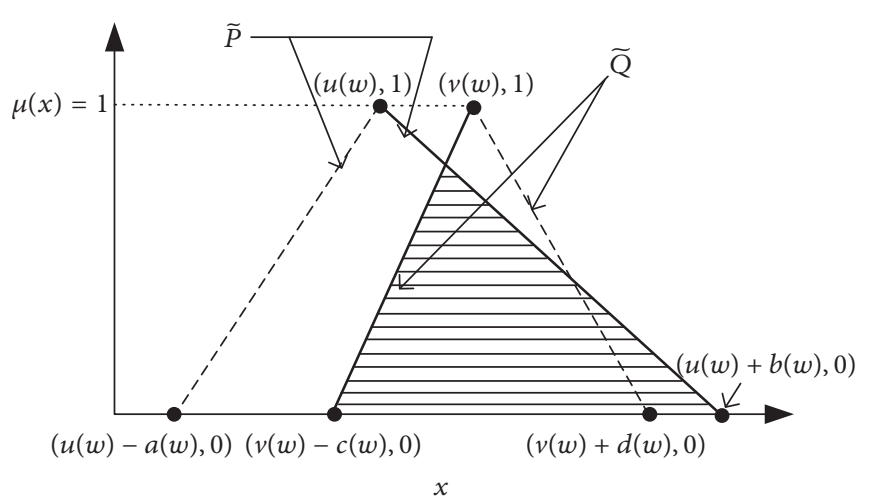

(c) Case 3

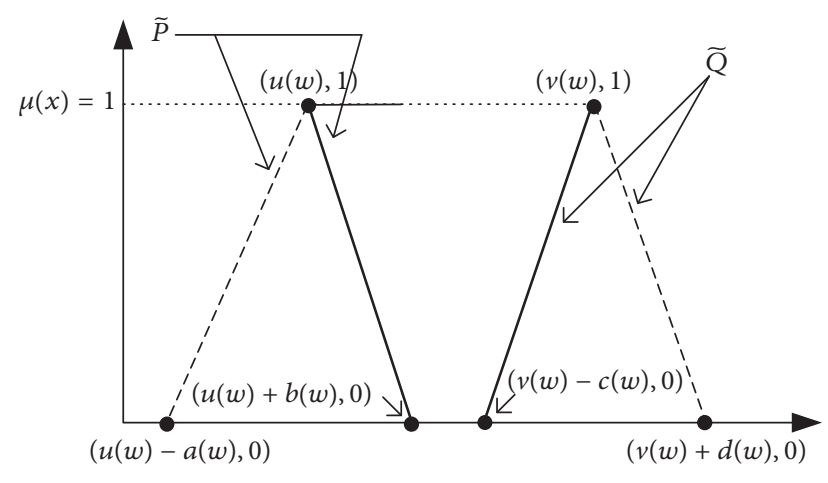

(e) Case 5

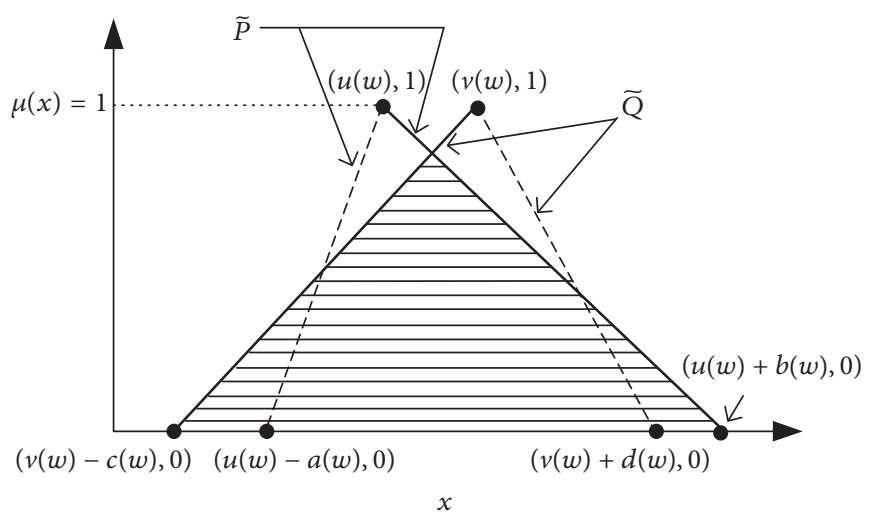

(g) Case 7

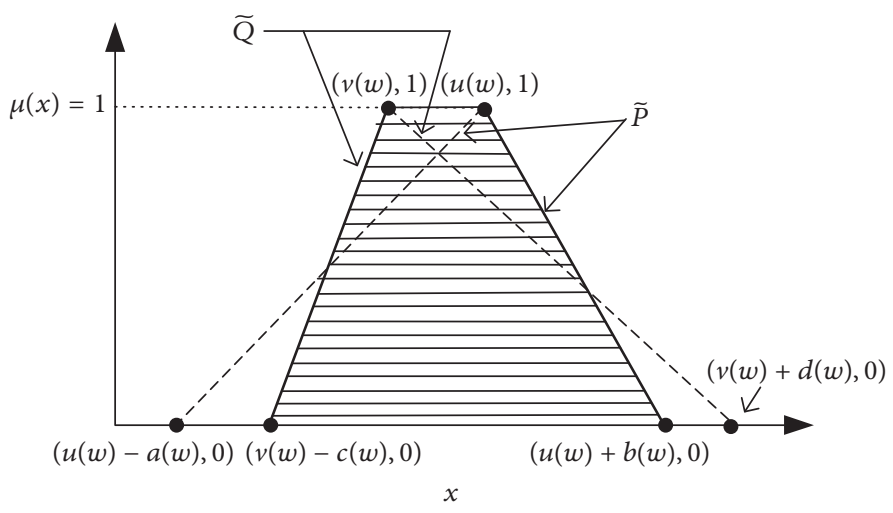

(b) Case 2

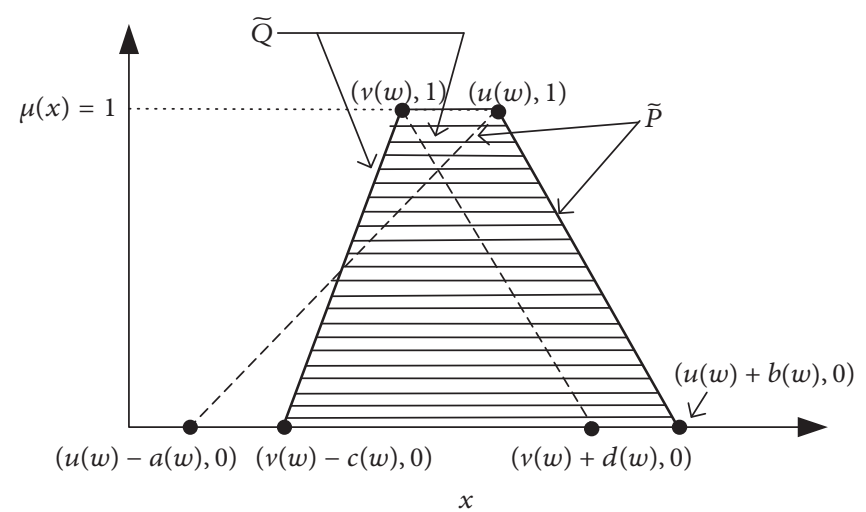

(d) Case 4

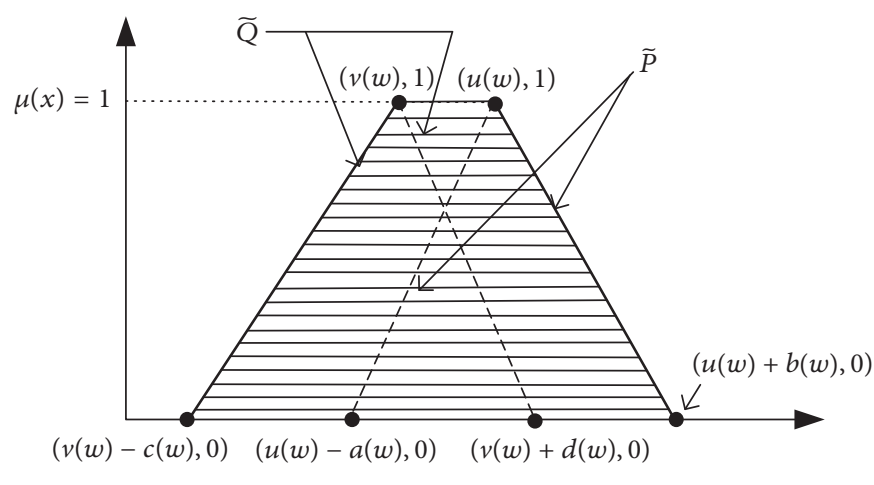

(f) Case 6

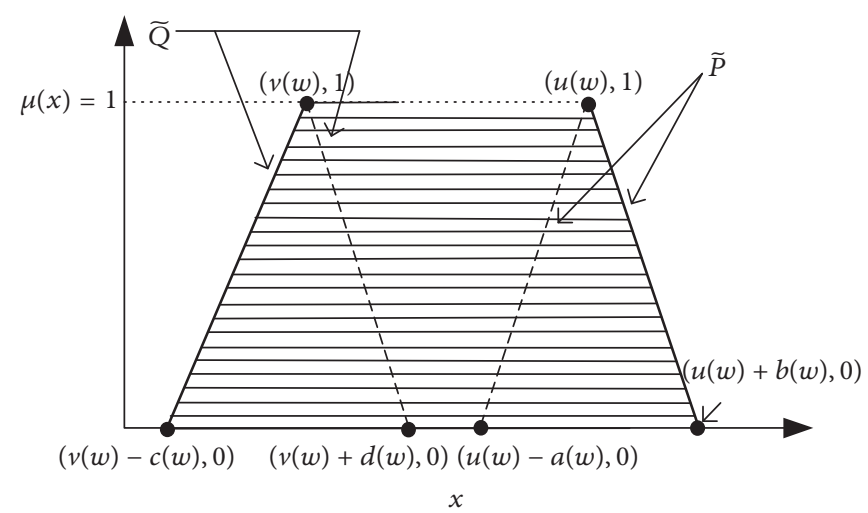

(h) Case 8

FIgUre 1: Continued. 


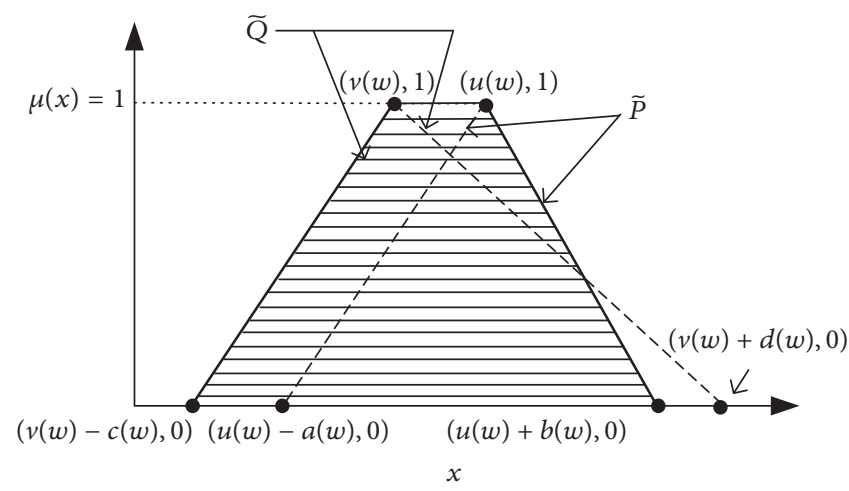

(i) Case 9

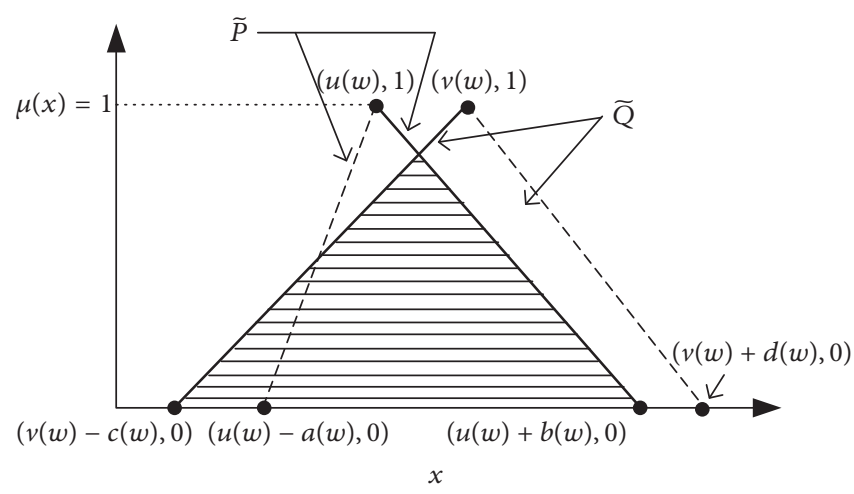

(j) Case 10

Figure 1

From our previous discussion, under the condition $u(w) \leq v(w)$, we find that

$$
\begin{aligned}
& \bar{D}(\tilde{\bar{P}}, \widetilde{\bar{Q}}) \\
& \quad=\max \left\{0, \frac{1}{2}[u(w)+b(w)-v(w)+c(w)]\right\} .
\end{aligned}
$$

On the other hand, under the condition $u(w)>v(w)$, we find that

$$
\bar{D}(\widetilde{\bar{P}}, \widetilde{\bar{Q}})=u(w)-v(w)+\frac{1}{2}[b(w)+c(w)]
$$

We summarize our findings in the next theorem.

Theorem 1 (revised version for Hop's Proposition 1, [12]). For two triangular fuzzy random numbers $\widetilde{\bar{P}}$ and $\widetilde{\bar{Q}}$, the average lower-side attainment of $\widetilde{\bar{P}}$ to $\widetilde{\bar{Q}}$ is derived as if $u(w) \leq v(w)$; then $\bar{D}(\widetilde{\bar{P}}, \widetilde{\bar{Q}})=\max \{0,(1 / 2)[u(w)+b(w)-v(w)+c(w)]\}$. Moreover, under the condition $u(w)>v(w)$, then

$$
\bar{D}(\tilde{\bar{P}}, \widetilde{\bar{Q}})=u(w)-v(w)+\frac{1}{2}[b(w)+c(w)]
$$

Remark 2. We may recall the proof of Hop [12] from his Proposition 1 to point out that he only considered Case 1 and subsequently overlooked the other cases.

Consequently, we revised Hop's Proposition 2 and recorded them in the next theorem.

Theorem 3 (revision for Proposition 2 of Hop [12]). For a given $w \in \Omega$, two triangular fuzzy random numbers $\widetilde{\bar{P}}$ and $\widetilde{\bar{Q}}$, with $\widetilde{P}_{w}=(u(w), a(w), b(w)), \widetilde{Q}_{w}=(v(w), c(w), d(w))$, with the scenario of $u(w) \leq v(w)$, then $\bar{V}(\widetilde{\bar{P}}, \widetilde{\bar{Q}}) \geq \lambda(w)$ if and only if

$$
\begin{aligned}
& \bar{D}(\tilde{\bar{P}}, \widetilde{\bar{Q}})=\max \left\{0, \frac{u(w)-v(w)+b(w)+c(w)}{2}\right\} \\
& \quad \geq \lambda(w), \\
& \bar{D}(\widetilde{\bar{Q}}, \tilde{\bar{P}})(w)=v(w)-u(w)+\frac{a(w)+d(w)}{2} \\
& \quad \geq \lambda(w) .
\end{aligned}
$$

For the scenario with $u(w)>v(w)$, then $\bar{V}(\tilde{\bar{P}}, \widetilde{\bar{Q}}) \geq \lambda(w)$ if and only if

$$
\begin{aligned}
& \bar{D}(\tilde{\bar{P}}, \widetilde{\bar{Q}})=\max \left\{0, \frac{u(w)-v(w)+b(w)+c(w)}{2}\right\} \\
& \quad \geq \lambda(w), \\
& \bar{D}(\widetilde{\bar{Q}}, \tilde{\bar{P}})(w)=v(w)-u(w)+\frac{a(w)+d(w)}{2} \\
& \quad \geq \lambda(w) .
\end{aligned}
$$

\section{Numerical Example}

To demonstrate the validity of our revision, we first consider the same numerical example as given in Hop [12] to look at the following fuzzy stochastic goal programming problem:

$$
\begin{array}{ll} 
& \widetilde{z}_{1}=\widetilde{c}_{11}(w) x_{1}+\widetilde{c}_{12}(w) x_{2} ; \widetilde{g}_{1}(w) \\
& \widetilde{z}_{2}=\widetilde{c}_{21}(w) x_{1}+\widetilde{c}_{22}(w) x_{2} ; \tilde{g}_{2}(w) \\
\text { s.t. } & \widetilde{\bar{A}} x \leq \tilde{\bar{b}} \\
& x \geq 0, \\
& w \in \Omega=\left\{w_{1}, w_{2}\right\}, \\
& p\left(w_{1}\right)=0.25, \\
& p\left(w_{2}\right)=0.75,
\end{array}
$$


where

$$
(\tilde{\bar{c}}, \widetilde{\bar{g}}, \widetilde{\bar{A}}, \tilde{\bar{b}})=\left\{\begin{array}{l}
\left(\widetilde{c}_{w 1}, \widetilde{g}_{w 1}, \widetilde{A}_{w 1}, \widetilde{b}_{w 1}\right)=\left\{\left(\begin{array}{ll}
\tilde{3} & \widetilde{4} \\
\widetilde{2} & \widetilde{3}
\end{array}\right),\left(\begin{array}{c}
\widetilde{10} \\
\widetilde{9}
\end{array}\right),\left(\begin{array}{cc}
\widetilde{1} & \tilde{1} \\
\widetilde{2} & \widetilde{1}
\end{array}\right),\left(\begin{array}{c}
\widetilde{3} \\
\widetilde{4}
\end{array}\right)\right\} \\
\left(\widetilde{c}_{w 2}, \widetilde{g}_{w 2}, \widetilde{A}_{w 2}, \widetilde{b}_{w 2}\right)=\left\{\left(\begin{array}{cc}
\widetilde{2} & \widetilde{1} \\
\widetilde{4} & \widetilde{3}
\end{array}\right),\left(\begin{array}{c}
\widetilde{8} \\
\widetilde{11}
\end{array}\right),\left(\begin{array}{cc}
\widetilde{1} & \widetilde{3} \\
\widetilde{1} & \widetilde{2}
\end{array}\right),\left(\begin{array}{c}
\widetilde{5} \\
\widetilde{4}
\end{array}\right)\right\}
\end{array}\right\}
$$

with the triangular fuzzy number denoted as $\widetilde{m}=(m, 1,1)$.

For the stochastic process with $w_{1}$, we need to find $\left(x_{1}, x_{2}\right)$ for the maximization problem with the constraints as shown below:

$\max \lambda_{1}\left(w_{1}\right)-\lambda_{2}\left(w_{1}\right)$

$$
\begin{aligned}
\text { Subject to } & \bar{D}\left(\left(\begin{array}{cc}
\widetilde{3} & \widetilde{4} \\
\widetilde{2} & \widetilde{3}
\end{array}\right)\left(\begin{array}{c}
x_{1} \\
x_{2}
\end{array}\right),\left(\begin{array}{c}
\widetilde{10} \\
\widetilde{9}
\end{array}\right)\right) \geq \lambda_{1}\left(w_{1}\right) \\
& \bar{D}\left(\left(\begin{array}{c}
\widetilde{10} \\
\widetilde{9}
\end{array}\right),\left(\begin{array}{cc}
\widetilde{3} & \widetilde{4} \\
\widetilde{2} & \widetilde{3}
\end{array}\right)\left(\begin{array}{l}
x_{1} \\
x_{2}
\end{array}\right)\right) \geq \lambda_{1}\left(w_{1}\right)
\end{aligned}
$$

$$
\bar{D}\left(\left(\begin{array}{ll}
\tilde{1} & \tilde{1} \\
\tilde{2} & \tilde{1}
\end{array}\right)\left(\begin{array}{l}
x_{1} \\
x_{2}
\end{array}\right),\left(\begin{array}{l}
\tilde{3} \\
\tilde{4}
\end{array}\right)\right) \leq \lambda_{2}\left(w_{1}\right)
$$

As $w_{1}$ does not contribute to the derivation and to simplify the expression, $w_{1}$ is omitted from $\left(\widetilde{3} x_{1}+\widetilde{4} x_{2}\right)\left(w_{1}\right)=\left(\left(3 x_{1}+\right.\right.$ $\left.\left.4 x_{2}\right)\left(w_{1}\right),\left(x_{1}+x_{2}\right)\left(w_{1}\right),\left(x_{1}+x_{2}\right)\left(w_{1}\right)\right)$ to result in a simpler expression $\widetilde{3} x_{1}+\widetilde{4} x_{2}=\left(3 x_{1}+4 x_{2}, x_{1}+x_{2}, x_{1}+x_{2}\right)$, which avoids ambiguity.

According to the revised Proposition 2, we employ the notation $B\left(w_{1}\right)$ and rewrite the (34) as follows:

$$
\begin{aligned}
& B\left(w_{1}\right)=\max \quad f\left(x_{1}, x_{2}\right) \\
& \text { Subject to } f\left(x_{1}, x_{2}\right)=\min \left\{A_{1}, A_{2}\right\}-\max \left\{A_{3}, A_{4}\right\} \\
& A_{1}= \begin{cases}\min \left\{\max \left\{0, \frac{4 x_{1}+5 x_{2}-9}{2}\right\}, \frac{21-5 x_{1}-7 x_{2}}{2}\right\}, & \text { if } 3 x_{1}+4 x_{2} \leq 10 \\
\min \left\{\frac{7 x_{1}+9 x_{2}-19}{2}, \max \left\{0, \frac{11-2 x_{1}-3 x_{2}}{2}\right\}\right\}, & \text { if } 3 x_{1}+4 x_{2}>10\end{cases} \\
& A_{2}= \begin{cases}\min \left\{\max \left\{0, \frac{3 x_{1}+4 x_{2}-8}{2}\right\}, \frac{19-3 x_{1}-5 x_{2}}{2}\right\}, & \text { if } 2 x_{1}+3 x_{2} \leq 9 \\
\min \left\{\frac{5 x_{1}+7 x_{2}-17}{2}, \max \left\{0, \frac{10-x_{1}-2 x_{2}}{2}\right\}\right\}, & \text { if } 2 x_{1}+3 x_{2}>9\end{cases} \\
& A_{3}= \begin{cases}\max \left\{0, \frac{2 x_{1}+2 x_{2}-2}{2}\right\}, & \text { if } x_{1}+x_{2} \leq 3 \\
\frac{3 x_{1}+3 x_{2}-5}{2}, & \text { if } x_{1}+x_{2}>3\end{cases} \\
& A_{4}= \begin{cases}\max \left\{0, \frac{3 x_{1}+2 x_{2}-3}{2}\right\}, & \text { if } 2 x_{1}+x_{2} \leq 4 \\
\frac{5 x_{1}+3 x_{2}-7}{2}, & \text { if } 2 x_{1}+x_{2}>4\end{cases}
\end{aligned}
$$

With a detailed analysis, it is found that $B\left(w_{1}\right)=0$ for all $\left(x_{1}, x_{2}\right)$ satisfying $x_{1} \geq 0, x_{2} \geq 0$ and $x_{1}+x_{2} \leq 1$. It is noted that the two values, the measures of the closeness, and the distance between two fuzzy numbers, in the objective functions, are placed with equal weights for the model. The relationship between them needs to be investigated in order to address the heterogeneous nature of the two measures.

Next, by considering the stochastic process with $w_{2}$, we locate $\left(x_{1}, x_{2}\right)$ for

$$
\begin{aligned}
& \text { Subject to } \bar{D}\left(\left(\begin{array}{cc}
\tilde{2} & \widetilde{1} \\
\widetilde{4} & \widetilde{3}
\end{array}\right)\left(\begin{array}{c}
x_{1} \\
x_{2}
\end{array}\right),\left(\begin{array}{c}
\widetilde{8} \\
\widetilde{11}
\end{array}\right)\right) \geq \lambda_{1}\left(w_{2}\right) \\
& \bar{D}\left(\left(\begin{array}{c}
\widetilde{8} \\
\widetilde{11}
\end{array}\right),\left(\begin{array}{cc}
\tilde{2} & \tilde{1} \\
\widetilde{4} & \widetilde{3}
\end{array}\right)\left(\begin{array}{l}
x_{1} \\
x_{2}
\end{array}\right)\right) \geq \lambda_{1}\left(w_{2}\right) \\
& \bar{D}\left(\left(\begin{array}{cc}
\tilde{1} & \widetilde{3} \\
\tilde{1} & \tilde{2}
\end{array}\right)\left(\begin{array}{l}
x_{1} \\
x_{2}
\end{array}\right),\left(\begin{array}{l}
\widetilde{5} \\
\tilde{4}
\end{array}\right)\right) \leq \lambda_{2}\left(w_{2}\right) \text {. }
\end{aligned}
$$


Again, based on revised Proposition 2, we employ the notation $B\left(w_{2}\right)$ and rewrite (41) as follows:

$$
\begin{aligned}
B\left(w_{2}\right)=\max & g\left(x_{1}, x_{2}\right) \\
\text { Subject to } & g\left(x_{1}, x_{2}\right)=\min \left\{A_{5}, A_{6}\right\}-\max \left\{A_{7}, A_{8}\right\} \\
A_{5} & = \begin{cases}\min \left\{\max \left\{0, \frac{3 x_{1}+2 x_{2}-7}{2}\right\}, \frac{17-3 x_{1}-x_{2}}{2}\right\}, \\
\min \left\{\frac{5 x_{1}+3 x_{2}-15}{2}, \max \left\{0, \frac{9-x_{1}}{2}\right\}\right\},\end{cases} \\
A_{6} & =\left\{\begin{array}{ll}
\min \left\{\max \left\{0, \frac{5 x_{1}+4 x_{2}-10}{2}\right\}, \frac{23-7 x_{1}-5 x_{2}}{2}\right\}, & \text { if } 2 x_{1}+x_{2}>8 \\
\min \left\{\frac{9 x_{1}+7 x_{2}-21}{2}, \max \left\{0, \frac{10-3 x_{1}-2 x_{2}}{2}\right\}, 3 x_{2} \leq 11\right.
\end{array}\right\}, \quad \text { if } 4 x_{1}+3 x_{2}>11 \\
A_{7} & = \begin{cases}\max \left\{0, \frac{2 x_{1}+4 x_{2}-4}{2}\right\}, & \text { if } x_{1}+3 x_{2} \leq 5 \\
\frac{3 x_{1}+7 x_{2}-9}{2}, & \text { if } x_{1}+3 x_{2}>5\end{cases} \\
A_{8} & = \begin{cases}\max \left\{0, \frac{2 x_{1}+3 x_{2}-3}{2}\right\}, & \text { if } x_{1}+2 x_{2} \leq 4 \\
\frac{3 x_{1}+5 x_{2}-7}{2}, & \text { if } x_{1}+2 x_{2}>4 .\end{cases}
\end{aligned}
$$

Again, the model yields the maximum that $B\left(w_{2}\right)=0$ for all $\left(x_{1}, x_{2}\right)$ satisfying $x_{1} \geq 0, x_{2} \geq 0$ and $2 x_{1}+3 x_{2} \leq 3$.

From the above discussion, we find that by considering the probability distribution $p\left(w_{1}\right)=0.25$ and $p\left(w_{2}\right)=0.75$, we can combine the stochastic processes of $w_{1}$ and $w_{2}$ to obtain

$$
\max f\left(x_{1}, x_{2}\right)+3 g\left(x_{1}, x_{2}\right)
$$

which is consistent with the probability distribution. According to our results with $B\left(w_{1}\right)=0$ and $B\left(w_{2}\right)=0$, it implies that the maximum value of $(48)$ is zero with $\left(x_{1}, x_{2}\right)$ satisfying $x_{1} \geq 0, x_{2} \geq 0$, and $x_{1}+x_{2} \leq 1$.

Recall Hop's solution of [12] with $\left(x_{1}, x_{2}\right)=(2.4,0)$ to imply $f(2.4,0)=-2.5, g(2.4,0)=-0.8$ and the objective value $f(2.4,0)+3 g(2.4,0)=-4$.9. It reveals that Hop [12] did not find the true maximum value, which is zero as obtained by our derivation.

Furthermore, we consider the penalty cost for violating the objective, $p=2$, and for the constraint, $p=3$. Eq. (36) and Eq. (43) can be revised as follows:

$$
\begin{aligned}
& 2 \min \left\{A_{1}, A_{2}\right\}-3 \max \left\{A_{3}, A_{4}\right\}, \\
& 2 \min \left\{A_{5}, A_{6}\right\}-3 \max \left\{A_{7}, A_{8}\right\} .
\end{aligned}
$$

The maximization problem of (48) is revised as

$$
\begin{aligned}
\max & 2 \min \left\{A_{1}, A_{2}\right\}-3 \max \left\{A_{3}, A_{4}\right\} \\
& +3\left[2 \min \left\{A_{5}, A_{6}\right\}-3 \max \left\{A_{7}, A_{8}\right\}\right] .
\end{aligned}
$$

Based on our results of $B\left(w_{1}\right)=0$ and $B\left(w_{2}\right)=0$, we rewrite the objective function in (50) as $2 B\left(w_{1}\right)+3 B\left(w_{2}\right)-$ $\max \left\{A_{3}, A_{4}\right\}-3 \max \left\{A_{7}, A_{8}\right\}$ such that the maximum value of (50) is thus zero for all $\left(x_{1}, x_{2}\right)$ satisfying $x_{1} \geq 0, x_{2} \geq 0$, and $x_{1}+x_{2} \leq 1$.

For completeness, we checked the results of Hop [12] with $\left(x_{1}, x_{2}\right)=(2.4,0)$ to find that the value of $(50)$ is -15 , which is not the maximum value as derived by ours.

Based on the above two numerical examples, if the proposition and solution procedures of Hop [12] are corrected, then, with the use of a modern computer, Hop [12] should derive the same (or at least, very close to) optimal value as ours. However, his results are far from the maximum value. This indicates that the Proposition in Hop [12] contains questionable results in the construction of the attainment index, and then our revision provides a patch work to enhance those results.

\section{Conclusion}

This paper provides a revision in response to the mathematical model presented by Hop. Hop aimed at the conversion of the fuzzy and stochastic LP model into a deterministic LP model through an approach that utilizes the attainment index. He emphasized that by carrying out the calculation of the attainment index, only a few additional constraints will be generated. However, the new approach which he developed does not guarantee finding the compromise solution since there exist several significant errors in his mathematical derivation and analytical results. This study has provided correction and improvement of his 
major procedure while simplifying the theoretical derivation processes.

\section{Conflicts of Interest}

The authors declare that there are no conflicts of interest regarding the publication of this paper.

\section{Acknowledgments}

The authors greatly appreciate the partially financial support of MOST 105-2410-H-015-006 and express their gratitude for the English revisions by Kaye Tanlee (kayetanlee@gmail .com).

\section{References}

[1] E. Czogala, "Multi-criteria decision making by means of fuzzy and probabilistic sets," Fuzzy Sets and Systems. An International Journal in Information Science and Engineering, vol. 36, no. 2, pp. 235-244, 1990.

[2] V. Mahalingam, N. Ranganathan, and J. E. Harlow, "A fuzzy optimization approach for variation aware power minimization during gate sizing," IEEE Transactions on Very Large Scale Integration (VLSI) Systems, vol. 16, no. 8, pp. 975-984, 2008.

[3] M. Inuiguchi and J. Ramik, "Possibilistic linear programming: a brief review of fuzzy mathematical programming and a comparison with stochastic programming in portfolio selection problem," Fuzzy Sets and Systems. An International Journal in Information Science and Engineering, vol. 111, no. 1, pp. 3-28, 2000.

[4] P. Fortemps and M. Roubens, "Ranking and defuzzification methods based on area compensation," Fuzzy Sets and Systems. An International Journal in Information Science and Engineering, vol. 82, no. 3, pp. 319-330, 1996.

[5] J. Chiang, "Fuzzy linear programming based on statistical confidence interval and interval-valued fuzzy set," European Journal of Operational Research, vol. 129, no. 1, pp. 65-86, 2001.

[6] K. D. Jamison and W. A. Lodwick, "Fuzzy linear programming using a penalty method," Fuzzy Sets and Systems. An International Journal in Information Science and Engineering, vol. 119, no. 1, pp. 97-110, 2001.

[7] H. J. Zimmermann, "Description \& optimization of fuzzy systems," International Journal of General Systems, vol. 2, no. 4, pp. 209-215, 1976.

[8] B. Liu, "Fuzzy random dependent-chance programming," IEEE Transactions on Fuzzy Systems, vol. 9, no. 5, pp. 721-726, 2001.

[9] M. K. Luhandjula, "Fuzziness and randomness in an optimization framework," Fuzzy Sets and Systems. An International Journal in Information Science and Engineering, vol. 77, no. 3, pp. 291-297, 1996.

[10] W. Fei, "Existence and uniqueness of solution for fuzzy random differential equations with non-Lipschitz coefficients," Information Sciences. An International Journal, vol. 177, no. 20, pp. 43294337, 2007.

[11] J. Xu and Y. Liu, "Multi-objective decision making model under fuzzy random environment and its application to inventory problems," Information Sciences, vol. 178, no. 14, pp. 2899-2914, 2008.
[12] N. V. Hop, "Fuzzy stochastic goal programming problems," European Journal of Operational Research, vol. 176, no. 1, pp. 7786, 2007.

[13] N. V. Hop, "Solving linear programming problems under fuzziness and randomness environment using attainment values," Information Sciences. An International Journal, vol. 177, no. 14, pp. 2971-2984, 2007.

[14] S.-Y. Chou, J. S.-J. Lin, and P. Julian, "Solving linear programming problems under fuzziness and randomness environment using attainment values," Information Sciences, vol. 179, pp. 4083-4088, 2009. 


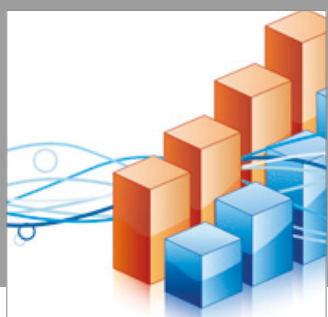

Advances in

Operations Research

vatersals

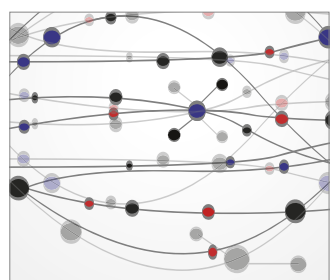

\section{The Scientific} World Journal
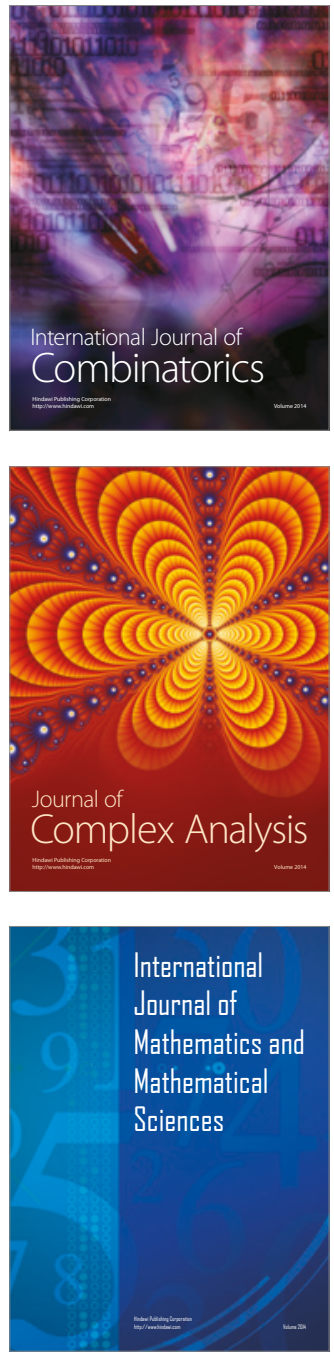
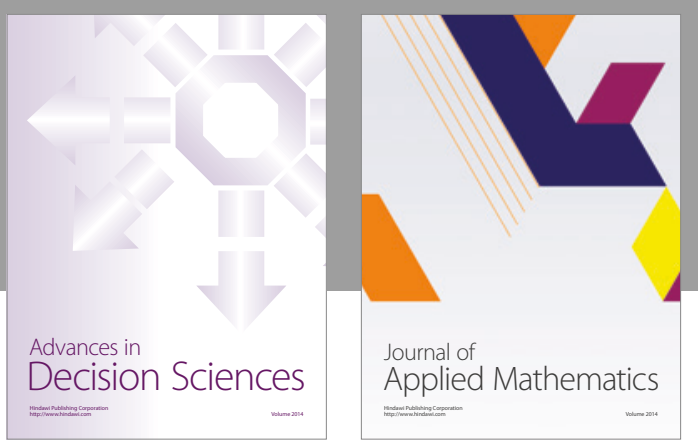

Algebra

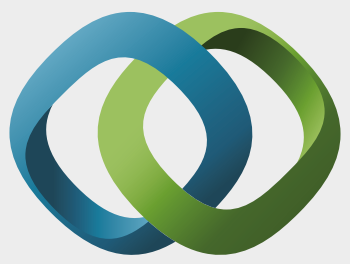

\section{Hindawi}

Submit your manuscripts at

https://www.hindawi.com
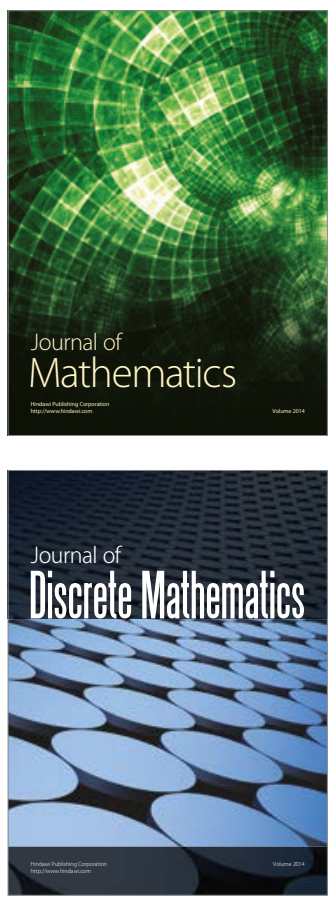

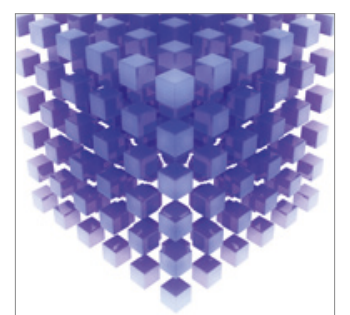

Mathematical Problems in Engineering
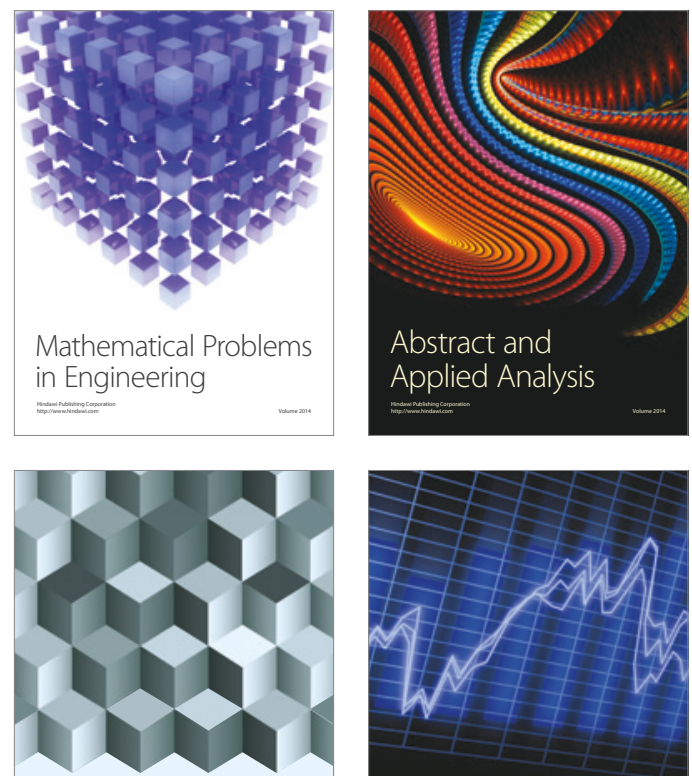

Journal of

Function Spaces

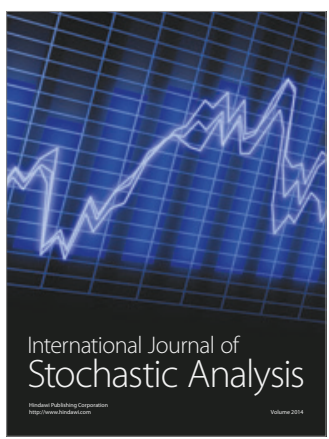

Probability and Statistics
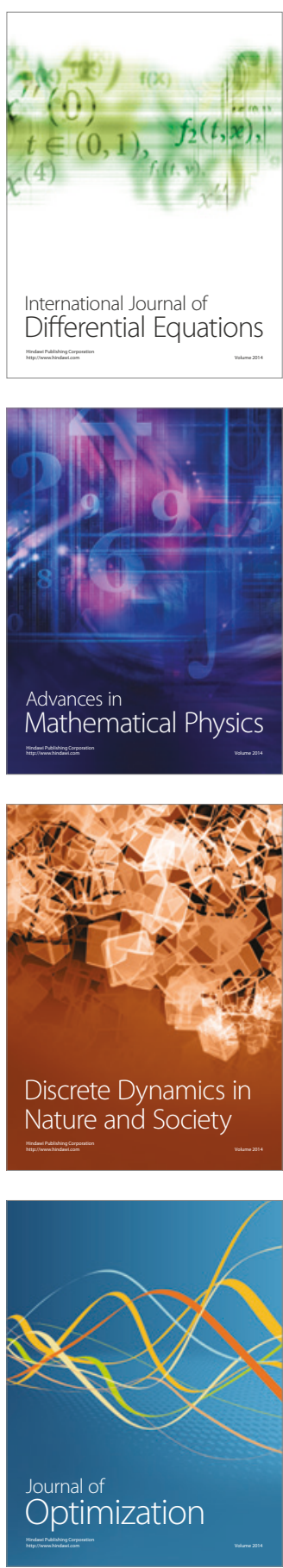